\title{
Smoke-Free Workplace Policies and Organizational Attraction
}

\author{
Hee Sun Park (Corresponding author) \\ Department of Communication, Michigan State University \\ East Lansing, MI 48824-1212, USA \\ Tel: 1-517-355-3480 E-mail: heesun@msu.edu \\ Seungcheol Austin Lee \\ Department of Communication, Michigan State University \\ East Lansing, MI 48824-1212, USA \\ E-mail: austiny@msu.edu \\ Seoyeon Hong \\ POSCO, Inc., Seoul, Korea \\ E-mail: hongseoy@msu.edu \\ Justin Cherry \\ International Academic Alliance \\ 250 W. 40th St. 10th Floor, New York, NY 10018, USA \\ E-mail: jcherry@iaaprograms.com \\ Youngyoul Fred Kang \\ Naver, Inc., Gyeonggi-do 463-844, Korea \\ E-mail: fred0830@gmail.com
}

Doshik Yun

Department of Communication, Michigan State University

East Lansing, MI 48824-1212, USA

E-mail: yundoshi@msu.edu

Hannah Klautke

Department of Communication, Michigan State University

East Lansing, MI 48824-1212, USA

E-mail: klautkeh@msu.edu

\begin{abstract}
Companies adopt smoke-free workplace policies to improve health of their employees, but how severely such policies are enforced can have an impact on non-smoking employees as well and can also affect employees' view about their companies. The current study examined the extent to which perceived severity of and organizational support for a smoke-free workplace policy affected employees' attraction toward their organizations. The data from 621 employees of 20 companies in the U.S. and 27 companies in Korea showed that the extent to which employees considered a smoke-free policy at their workplace to be enforced severely was negatively related to organizational attraction (coefficient $=-0.22, p=.002$ ) and perceived organizational support was positively related to organizational attraction (coefficient $=0.41, p<.001$ ). The negative relationship between perceived
\end{abstract}


severity and organizational attraction, however, became weaker for organizations that had employees with higher perceptions of organizational support. In contrast to smokers (coefficient $=-.05$ ), ex-smokers' perceived severity of a smoke-free policy was positively related to their organizational attraction (coefficient $=.31$ ). These findings indicated that a smoke-free policy in the workplace can have implications for non-smokers, including ex-smokers, as well as for smokers.

Keywords: Smokers, Smoke-free policy, Organizational attraction, Perceived organizational support, Korea, The United States of America

\section{Introduction}

Smoking, like many other behaviors associated with increased health risk, is no longer a personal matter. As healthcare costs continue to rise, employers are taking preventive action, monitoring, and even restricting employees' off-duty behaviors that do not coincide with employers' interests (Anderson, 2003). Smoking is one of the non-healthy behaviors that organizations frequently regulate. Besides making employees healthier, however, a health policy has the potential to strengthen or weaken employees' relationships with the organization impacting organizational attractiveness, satisfaction, work effort and turnover intention (Goetzel \& Ozminkowski, 2000). A workplace policy regarding a health matter may result in undesirable outcomes, such as employees' negative view of the organization or bad publicity (Zoller, 2004).

A smoke-free policy, although directly relevant to employees who smoke, can have implications that extend beyond the susceptible group to be of concern to all employees, including non-smokers and ex-smokers who managed to quit. The factors that potentially influence the effects of a smoke-free policy therefore warrant a closer look. Thus, the current study focused on how employees' attraction toward their attraction would be affected by three factors: employee perceptions of the severity of their organization's smoke-free policy, employee perceptions of organizational support available, and individual employees' smoking status.

\subsection{Smoke-Free Policies}

As a leading cause of preventable illnesses and premature deaths, increasing the risk for heart disease, stroke, emphysema, and many cancers, smoking is responsible for about 438,000 deaths annually in the U.S. (Center for Disease Control and Prevention, 2005). Direct costs to businesses associated with smoking reach $\$ 75$ billion a year in direct medical costs, $\$ 92$ billion in lost productivity, and $\$ 10$ billion in exposure to second-hand smoke (Meritain Health, 2007; Osinubi, Barbeau, Williams, \& Sorensen, 2005). In Korea, the annual social cost caused by smoking was estimated to be $\$ 136$ per capita (Ji, 2003).

As early as 1984, 56\% of the Fortune 500 and many smaller companies had instituted some type of smoking cessation support program, and twenty years later, almost $83 \%$ of health promotion programs involved a smoking cessation component (Harris, 1994). Companies in Korea also have taken measures to control employee smoking. For example, SK Telecom offers a smoking cessation fund, which includes monthly nicotine testing and provides monetary rewards for successful smoking cessation (M. Kim, 2008). One of Samsung's company worksites has banned smoking in the entire worksite, replaced all the three designated smoking areas with work-out areas, and prohibited bringing any cigarettes and lighters to the worksite (Lee, 2009). Recently, the CEO of POSCO in Korea announced his plans to encourage all employees to quit smoking by the end of 2009 and to administer blood tests to see if any employees smoke at home (Y.-M. Kim, 2009). Whether or not support for smokers is being offered, programs designed to eliminate or regulate smoking at the worksite continue to gain in prevalence (Sofian, McAfee, Doctor, \& Carson, 1994).

Employers can take proactive steps to encourage employees' adoption of healthy habits. Enforcing a smoke-free workforce policy, however, poses a dilemma for organizations. Organizations may maintain a smoke-free workplace (i.e., restricting employees' smoking to a designated area or prohibiting smoking on the organization's premises) or a smoke-free workforce (i.e., firing smokers or hiring only non-smokers). They may also take various approaches in between, such as using incentive schemes to encourage employers to quit. There may be hidden costs of negative responses to restrictive smoke-free policies: invasion-of-privacy concerns (especially in cases where nicotine testing is employed), reduced performance, and negative employee attitudes as they were reported in the context of employee drug testing (e.g., Konovski \& Cropanzano, 1991).

\subsection{Organizational Attraction}

Organizational attraction is based on employees' perception of compatibility, or a match between their personalities, attitudes, and values and the organization's values, goals, structures, processes, and cultures (Chatman, 1991; Schneider, Goldstein, \& Smith, 1995). Organizational attraction is characterized as individuals' assessment of an organization as a good place to work (Highhouse, Lievens, \& Sinar, 2003). In its introduction 
or maintenance of a smoke-free policy, an organization may have diverse goals and values such as promoting employees' health, increasing productivity at work, and saving on health-care costs. The degree of match or mismatch between employees' behaviors and the organizational goals may affect employees' attraction to their organizations.

\subsection{Perceived Severity}

Some organization may have a severe policy by imposing harsh penalties (e.g., employment termination) for non-compliance from employees, whereas others may take a more lenient approach and uses a non-severe policy by simply encouraging voluntary participation from employees. In the case of alcohol testing and treatment, people felt a higher level of fairness and organizational attraction when an organizational policy was presented as voluntary than when the policy was described as a coercive or monitoring requirement (Truxillo, Bauer, \& Paronto, 2002). For a smoke-free policy, employees may also perceive a severe health policy as the implementation of unfair restrictions and infringements on their own rights as individuals, and thus decrease attraction to their organization. Dalsey and Park (2009) showed that the extent to which job applicants lowered their organizational attraction toward an organization was greater for an organization with a high-severity smoke-free policy than an organization with a low-severity smoke-free policy. Thus, it is hypothesized that individuals' perception about the severity of smoke-free policy will be negatively related to organizational attraction (H1).

\subsection{Perceived Organizational Support}

Employees' perception of organizational support can also be related to the level of attraction employees have toward their organization. An organization can demonstrate that it genuinely cares about its employees' health by introducing or maintaining a health program involving a great deal of assistance, and employees may have a favorable evaluation of their organization for its efforts. Research also found a positive relationship between perceived organizational support and organizational commitment, job satisfaction, positive moods at work, and intentions to stay in the organization (Rhoades \& Eisenberger, 2002). Thus, it is hypothesized that employees' perception regarding how much support their organization provides them in relation to smoke-free policy will be positively related to their organizational attraction $(\mathrm{H} 2)$.

\subsection{Smoking Status}

Although perceived severity and perceived organizational support may independently affect organizational attraction, their effect may vary depending on employees' smoking status. Smoking employees may decrease organizational attraction to a greater extent as they are more likely to perceive a smoke-free policy as severe because their smoking behaviors are not consistent with organizational values and goals. On the other hand, non-smokers and ex-smokers may increase their organizational attraction because they find their concern for health consistent with that of their organization. Thus, although employees may perceive a smoke-free policy as severe to a similar extent, they may exhibit a different level of organizational attraction depending on their smoking status. Thus, it is asked how will the effect of perceived severity on organizational attraction vary for smokers, ex-smokers, and non-smokers (RQ1).

Smoking status may be also responsible for the differential effect of perceived organizational support on organizational attraction. It is possible that smokers are more likely to appreciate organizational support than non-smokers because they are the actual beneficiaries of the support. However, non-smoking employees may also increase their organizational attraction as much as smoking employees may because, although they do not receive organizational support in relation to a smoke-free policy, they may regard organizational concerns for its employees as consistent with their values. In addition, ex-smokers may be more likely to rely on perceived organizational support in deciding on the level of organizational attraction than smokers. The former may feel greater necessity of organizational support than the latter because of their past smoking habits and efforts to continue smoking cessation. To explore the differential effect of perceived organizational support on organizational attraction, it is asked how will the effect of perceived organizational support (POS) on organizational attraction vary for smokers, ex-smokers, and non-smokers (RQ2).

\section{Method}

\subsection{Participants}

Participants were 235 employees from 20 companies in the U.S. and 386 employees from 27 companies in Korea. The companies encompassed diverse industries such as health care, technology, public service, finance, education, and manufacturing in both countries. Of U.S. participants, $93 \%$ were full time employees (age $M=$ $36.26, S D=11.40,65.2 \%$ women). In addition, Caucasians comprised $81.3 \%$ of the participants, African 
Americans 4.3\%, Hispanics 4.3\%, Native Americans 1.7\%, and others 8.4\%. Lastly, 35.7\% participants had an entry-level position, $21.7 \%$ had a mid-management level position, $4.3 \%$ had a senior-management level, and $38.0 \%$ had positions other than those mentioned above. Of Korean participants, $88.3 \%$ were full time employees (age $M=32.35, S D=7.85,39.5 \%$ women). All Korean participants were ethnically Korean. Lastly, 51.8\% participants had an entry-level position, $32.9 \%$ had a mid-management level position, $6.7 \%$ had a senior-management level, and $8.6 \%$ had positions other than those mentioned above.

\subsection{Procedure}

Companies located in Michigan and Utah in the U.S. and companies located in Seoul and Busan in Korea were contacted randomly or via personal ties. Once companies agreed to participate in the study, a researcher visited each company to distribute the survey questionnaire to those willing to participate. Participants filled out the survey in a place of their choices (e.g., a conference room or a coffee room) located in the company. The average time for the completion of the survey was about ten minutes.

\subsection{Measures}

The questionnaire was first produced in English and then translated to Korean. The English version of the questionnaire was compared with Korean versions, using the community translation method (i.e., four individuals carefully translated each item together and resolved any discrepancies in group discussion) to ensure equivalence in meaning. Participants completed the questionnaire in their native language. The measures used a 7-point response format (e.g., $1=$ strongly disagree, $7=$ strongly agree). Given the necessity of minimizing the questionnaire length for working adults, a small number of measurement items were selected for each variable based on high item-total correlations from a past study (Dalsey \& Park, 2009). Confirmatory factor analysis (CFA) and reliability analysis conducted on the current data showed an acceptable level of measurement validity as explained below.

Confirmatory factor analysis (CFA) was performed on the measures for organizational attraction, perceived severity, and perceived organizational support to see if these measures were indicators of three separate factors and also to examine measurement equivalence. A multiple group CFA analysis showed that the three-factor model was a good fit even when the factor loadings and factor covariances were constrained equal across US and Korean data (CFI [Comparative Fit Index] $=.95$, TLI [Tucker-Lewis Index] $=.95$, RMSEA [Root Mean Square Error of Approximation] $=.059$, SRMR [standardized root mean residual $]=.076$ ).

\subsubsection{Organizational Attraction}

Organizational attraction was measured by three items (Cronbach's $\alpha=.92$ in the U.S. and .88 in Korea) adapted from Highhouse et al.'s (2003) organizational attraction scale and one additional item created for this study. Items were "For me, my current workplace is a great place to work," "Employees are proud to say they work at my current workplace," and "My current workplace has a reputation as being an excellent employer." The additional item asked participants to circle the expression that best characterizes their level of satisfaction with working at their current workplace on a 7 -point scale $(1=$ not at all satisfied and $7=$ most satisfied $)$.

\subsubsection{Perceived Severity}

Perceived severity was measured with three items ( $\alpha=.77$ in the U.S. and .71 in Korea). Items were "I believe adherence to the non-smoking policy is required to maintain employment," "I believe employment is contingent upon adherence to the non-smoking policy," and "I believe the non-smoking policy is serious to smokers' employment."

\subsubsection{Perceived Organizational Support}

Six items ( $\alpha=.92$ in the U.S. and .89 in Korea), modified from the Survey of Perceived Organizational Support (POS) (Eisenberger, Huntington, Hutchison, \& Sowa, 1986; Eisenberger, Armeli, Rexwinkel, Lynch, \& Rhoades, 2001), were used to measure perceived organizational support in the current study. Example items included "my current company assists in helping employees quit smoking" and "my current company is sincere in its effort to help employees."

\subsubsection{Statistical Analysis}

The data were analyzed with Hierarchical Linear Modeling (HLM) (Raudenbush \& Bryk, 2002) because individual employees were nested in their respective organization. The multilevel analysis allowed for partitioning of variance in the individual-level dependent variable, organizational attraction, into between-individual (i.e., individual-level, level-1) and between-organization (i.e., organization-level, level-2) components. The individual-level predictors were group-mean-centered for proper testing of cross-level 
interaction (Park, 2008; Raudenbush \& Bryk, 2002), and organization averages of perceived severity and perceived organizational support were included as level-2 predictors. Smoker status was converted into two dummy variables: ex-smoker and non-smoker. For the ex-smoker variable, smokers were coded as 0 (the reference group) and ex-smokers were coded as 1 (the comparison group). For the non-smoker variable, smokers were coded as 0 and non-smokers as 1 . Nation was dummy-coded with U.S. as 0 and Korea as 1 . For individual level interaction terms, perceived severity and perceived organizational support were grand-mean-centered before being multiplied with each of the dummy variables. Table 1 shows the result of HLM analysis.

\section{Results}

\subsection{Preliminary Analyses}

Organization-level variance in organizational attraction was significant, variance $=0.59, \chi^{2}(46)=424.28, p$ $<.001$. Intraclass correlation (ICC) for organizational attraction was .39, indicating that $39 \%$ of the variance in organizational attraction was between organizations and $61 \%$ was between individuals. For smoking status, $23.8 \%$ of U.S. participants identified themselves as smokers, $22.1 \%$ as ex-smokers, and $54.0 \%$ as non-smokers. Of Korean participants, $30.3 \%$ identified themselves as smokers, $12.2 \%$ as ex-smokers, and $57.0 \%$ as non-smokers. Two dummy variables indicating smoking status was level-1 predictors. Organization-level variance in perceived organizational support was significant, variance $=0.68, \chi^{2}(46)=433.21, p<.001$, and substantial with ICC of .40 . Because the organization-level variance in perceived organizational support was substantial, perceived organizational support was treated as both an individual-level (level-1) predictor and an organization-level (level-2) predictor in the main analysis. Organization-level variance in perceived severity was significant, variance $=0.07, \chi^{2}(46)=70.16, p=.012$, but not substantial, because ICC was .03 . Because the organizational-level (level-2) variance in perceived severity was not large, perceived severity was mainly treated as an individual-level predictor in the main analysis.

For employees in the U.S., perceived severity was positively correlated to perceived organizational support, $r$ $(233)=.16, p=.016$, and also positively correlated to organizational attraction, $r(233)=.13, p=.04$. Perceived organizational support was positively correlated to organizational attraction, $r(233)=.62, p<.001$. For employees in Korea, perceived severity was positively correlated to perceived organizational support, $r$ (381) $=.10, p=.045$, but was not significantly correlated to organizational attraction, $r(381)=.08, p=.14$. Perceived organizational support was positively correlated to organizational attraction, $r(381)=.53, p<.001$. Americans $(M=3.90, S D=1.56)$ reported higher perceived severity than Koreans $(M=3.54, S D=1.35), t(616)=2.98, p$ $=.003$. Americans $(M=4.94, S D=1.35)$ reported higher perceived organizational support than Koreans $(M=$ $3.50, S D=1.08), t(616)=14.66, p<.001$. Americans $(M=5.86, S D=1.06)$ reported higher organizational attraction than Koreans $(M=4.63, S D=1.08), t(616)=13.75, p<.001$.

\subsection{Main Analysis}

\subsubsection{Level-1 Predictors}

Including the two level-1 predictors in the model explained $39.23 \%$ of the individual-level variance in organizational attraction. As shown in Table 1, perceived severity was a significant and negative predictor (coefficient $=-0.22, p=.002$ ) and perceived organizational support was a significant and positive predictor (coefficient $=0.41, p<.001)$.

For individual-level interaction terms, the product term of ex-smoker by perceived severity (coefficient $=0.21, p$ $=.016$ ) and the product term of non-smoker by perceived severity (coefficient $=0.13, p=.034$ ) were significant and positive. For the purpose of clarifying the interaction patterns, ordinary least square multiple regression analyses were done separately for non-smokers, ex-smokers, and smokers. These findings indicated that the relationship between perceived severity and organizational attraction was positive among ex-smokers $(\beta=.31)$ and non-smoker $(\beta=.08)$, whereas it was negative among smokers $(\beta=-.05)$.

As shown in Table 1, the product term of ex-smoker by perceived organizational support (coefficient $=0.13, p$ $=.115$ ), the product term of non-smoker by perceived organizational support (coefficient $=0.10, p=.110$ ), and the product term of perceived severity by perceived organizational support (coefficient $=-0.02, p=.545$ ) were not significant in explaining organizational attraction.

The analysis further showed that the variance in level-1 intercept was significant, variance $=0.21, \chi^{2}(44)=$ $222.49, p<.001$, indicating that the group average organizational attraction varied across organizations, and that level-2 predictors were necessary to explain the variance. The variance in level-1 slope for perceived severity was significant, variance $=0.013, \chi^{2}(46)=65.20, p=.032$, indicating that the effect of perceived severity on organizational attraction varied across organizations, and that level-2 predictors were necessary to explain the 
variance in the slope of perceived severity. In addition, the variance in level-1 slope for perceived organizational support was significant, variance $=0.06, \chi^{2}(46)=82.89, p<.05$, indicating that the effect of perceived organizational support on organizational attraction varied across organizations, and that level-2 predictors could be used to explain which companies had a stronger or weaker effect of perceived organizational support on organizational attraction.

None of the level-1 interaction term predictors had a significant variance in their slopes, indicating that no level-2 predictors were necessary to explain the variance in the slopes of these five level-1 interaction terms. The slope of the interaction term of ex-smoker by perceived severity (variance $\left.=0.02, \chi^{2}[24]=13.71, p>.50\right)$, the slope of the interaction term of ex-smoker by perceived organizational support (variance $=0.09, \chi^{2}[24]=34.56$, $p=.08)$, the slope of the interaction term of non-smoker by perceived severity (variance $=0.03, \chi^{2}[24]=25.15$, $p=.40$ ), the slope of the interaction term of non-smoker by perceived organizational support (variance $=0.02, \chi^{2}$ $[24]=22.03, p>.50)$, and the slope of the interaction term of perceived severity by perceived organizational support (variance $=0.01, \chi^{2}[24]=30.86, p=.16$ ) did not vary significantly across organizations.

\subsubsection{Level-2 Predictors}

As shown in Table 1, when organizational average of perceived severity and that of perceived organizational support were included as organization-level (i.e., level-2) predictors of the variance in level-1 intercept (i.e., organizational average of organizational attraction), organizational average of perceived severity was not significant (coefficient $=0.21, p=.215$ ), but organizational average of perceived organizational support was significant in explaining organizational average of organizational attraction (coefficient $=0.31, p=.011$ ). Nationality was a significant predictor of organizational average of organizational attraction (coefficient $=-0.67$, $p=.001$ ), indicating that employees of companies in the U.S. had higher organizational attraction than did those of companies in Korea. The three level-2 predictors (nationality, organizational averages of perceived severity, and perceived organizational support) together explained 73.95\% of the variance in level-1 intercept (i.e., organizational average of organizational attraction). Among these three level-2 predictors, nation was the strongest predictor, explaining $65.38 \%$ of the variance in 1 organizational average of organizational attraction.

Additionally, when nation was included as a level-2 predictor of the variance in the level-1 slope of perceived severity, it was not significant (coefficient $=0.15, p=.053$ ). On the other hand, when organization average of perceived organizational support was included as an organization-level (i.e., level-2) predictor of the variance in the level-1 slope of perceived severity, it was significant (coefficient $=0.13, p=.013$ ), indicating that the negative relationship between perceived severity and organizational attraction became weaker for organizations that had employees with a higher perception of organizational support. Perceived organizational support as a level-2 predictor accounted for $11.60 \%$ of the variance in the level-1 slope of perceived severity.

As mentioned above, the effect of perceived organizational support on organizational attraction varied across organizations. Nationality was not a significant predictor of variance in the slope of perceived organizational support (coefficient $=-0.12, p=.257$ ), indicating that there were no substantial cross-national differences in how perceived organizational support was related to organizational attraction.

\section{Discussion}

This study found that an increase in perceived severity of a smoke-free policy was associated with lower organizational attraction. Another interesting finding about perceived severity was that there were much larger variances among individuals regarding their perception of the policy's severity than among organizations. That is, even among those who worked for the same company, no substantial amount of consensus existed about how severely a smoke-free policy was enforced in their organization. It is likely that a host of moderating factors, such as procedural justice characteristics of the inception, communication, and enforcement of the policy, or individual health values and orientations can lead to variation in how severe the same smoking policy is perceived to be by different employees. Regardless of what the actual content of smoke-free policy is in any given organization, it is this perceived severity that matters for subsequent outcomes, such as organizational attraction.

The current finding showed a positive relationship between perceived organizational support and organizational attraction. The current paper characterized perceived organizational support as a sense of goodwill on the part of the company towards its employees, in terms of providing assistance for both smoking cessation efforts and the general welfare of employees. Perceived organizational support had been shown previously to outweigh policy severity differences in its influence on organizational attraction outcomes (Dalsey \& Park, 2009). The feeling that "this company truly cares about me," as opposed to "this company simply wants me to function like a non-smoking cog in the machine," seems to strengthen employees' affinity towards the organization; this may be 
based on a norm of reciprocity that not only serves to enhance positive moods at work, but also increases employees' willingness to comply with company policies (Konovski \& Cropanzano, 1991; Rhoades \& Eisenberger, 2002). Considering that the positive effect of perceived organizational support on organizational attraction was consistent across smokers, ex-smokers, and non-smokers, this finding may imply that providing care and assistance to smokers can also have positive effects on non-smoking employees.

Additionally, the current study found that perceived organizational support moderated the effect of perceived severity on organizational attraction. The finding may indicate that when an organization provides highly supportive assistance beneficial to employees, severity of a smoke-free policy will have less negative impact on attraction toward organizations than when an organization does not provide any assistance or provides assistance perceived as non-beneficial to employees. Considering the importance of perceived organizational support for its effect on organizational attraction, one needs to consider the possibility that, for a given organizational assistance program, employees may differ from top management in their evaluation of how supportive the assistance program is to employees. That is, what top management considers as supportive and beneficial to employees may not be perceived likewise by those employees. Thus, when an organization provides highly supportive assistance that the targeted employees also consider beneficial, the organization may able to implement a highly severe smoke-free policy seriously and avoid undesirable attribution about the reason for its smoke-free policy (e.g., the organizational only cares about money and wants to fire smokers to reduce its health care costs.).

\subsection{An Important Moderator: Smoking Status}

The effects of perceived severity on organizational attraction varied for smokers, ex-smokers, and non-smokers. More so than in the case of non-smokers, high perceived severity was associated with high organizational attraction for ex-smokers. This finding is consistent with another study (Park, Dalsey, Kang, Hong, \& Lee, in press) in that Korean ex-smoker undergraduates showed greater organizational attraction toward companies with a highly severe smoke-free policy than companies with a low severity policy. It is possible that ex-smokers possess key qualities that lead them to valuing smoke-free policies: they are likely to hold negative views on smoking and believe in the seriousness of its consequences on one's health, and, having successfully gone through the process of quitting, are likely to hold efficacy beliefs about their own, but also other people's ability to quit. A severe policy forcing the employee to do the 'right' thing is likely to be perceived as a valuable tool for personal improvement, rather than an unfair threat or punishment for some behaviors beyond the individual's control. The latter is a likely perception of severe policies by smokers, which may serve to explain the negative effect of perceived severity on organizational attraction by that group. Consistent with the proposed explanations, the effect of perceived severity was nearly zero among non-smokers. This indifference toward the policy in part certainly reflects a lack of personal involvement in the issue, but may also be rooted in averaged-out effects of concern for privacy on one hand and a fit between individual and organizational values on the other.

\subsection{Practical Implications}

First, when introducing a new smoke-free policy or maintaining the current smoke-free policy, an organization needs to be concerned with the level of severity of the policy and organizational support to maintain its organizational attraction among its employees. When an organization needs to keep a high level of severity of a smoke-free policy, it should provide justification for its policy so that employees will not perceive the smoke-free policy as severe. In addition, an organization needs to provide tangential support for smoking employees in relation to a smoke-free policy. Once employees regard their organization as caring for employees' health, an organization may prevent many negative consequences of a smoke-free policy.

Second, an organization needs to be aware of the fact that a smoke-free policy in the workplace also has implications for non-smokers, including ex-smokers as well as smokers. The finding that severity decreased organizational attraction among smokers but increased organizational attraction among ex-smokers implies that an organization needs to decide which group of employees constitutes a larger or more vital workforce to the organization and base the level of severity on the assessment. In addition, given that organizational support was related to organizational attraction across all types of employees, an organization also needs to make an effort in communicating its support for smoking cessation efforts and a smoke-free policy to non-smoking employees as well as smoking employees.

Third, although there were national differences between the U.S. and Korea in their average scores on organizational attraction, perceived severity, and perceived organizational support, the positive and negative relationships among organizational attraction, perceived severity, perceived organizational support, and smoking status were highly similar for both nations. This finding may provide implications for multinational corporations in such a way that, at least in the case of smoke-free policies in the U.S. and Korea, employers' genuine concern 
for employees' health can have universal positive effects.

\subsection{Limitations}

The current study has two major limitations. First, since participants were recruited from only 47 organizations, it did not provide sufficient variances to examine the effects of diverse organizational level variables, such as industry type and organization size. It might be that employees in health related industries had higher organizational attraction as severity of their organization's smoke-free policy increased because of their interests in and concerns about health issues. Thus, future research should be directed towards including organizational level variables by soliciting participation from many organizations in diverse industries.

Second, since employees participated in the current study on a voluntary basis and via convenient sampling, it is not certain whether those who agreed to participate were representative members of organizations. It could be argued that results from the current study were biased because only those who were interested in smoke-free policy issues might have participated in the current study.

Third, the current findings were based on individuals' self-reported responses and perceptions about smoke-free policies and their organizations. Additionally, because the data were collected with a single survey at a single point in time, there can be issues concerning common method variance. Future studies may consider including more objective or formal characteristics of smoke-free policies and organizational support to see how these objective features and employees' subjective perceptions are consistent with one another and whether they affect similarly or differentially employees' views about their organizations.

\section{Conclusion}

Policies and programs that organizations implement can be relevant to not only the targeted groups of employees but also others to whom the policies and programs are supposed to be irrelevant. As shown in this study, ex-smoker employees' perception of smoke-free policy severity was positively related to organizational attraction, whereas smoking employees' perception of smoke-free policy severity was negatively related to organizational attraction. Additionally, for all of non-smoker, ex-smoker, and smoker employees, perceived organizational support provided for smoking cessation efforts was positively related to organizational attraction. As many organizations may start implementing various health policies, it may be interesting to see if the current findings can generalize to the targeted and non-targeted employees of health policies other than smoke-free policies.

\section{References}

Anderson, D. R. (2003). Building a first workforce: The sky's the limit with population health Management. Absolute Advantage, 2, 4-9.

Center for Disease Control and Prevention. (2005). Annual smoking - attributable mortality, years of potential life lost, and productivity losses - United States, 1997-2001. Morbidity and Mortality Weekly Report 2005, 54, 625-628. Retrieved on April 23, 2006. [Online] Available: http://www.cdc.gov/mmwr/preview/mmwrhtml/mm5425a1.htm

Chatman, J. A. (1991). Matching people and organizations: Selection and socialization in public accounting firms. Administrative Science Quarterly, 36, 459-484.

Dalsey, E., \& Park, H. S. (2009). Implication of organizational health policy on organizational attractiveness. Health Communication, 24, 71-81.

Eisenberger, R., Armeli, S., Rexwinkel, B., Lynch, P. D., Rhodes, L. (2001). Reciprocation and perceived organizational support. Journal of Applied Psychology, 86, $42-57$.

Eisenberger, R., Huntington, R., Hutchison, S., \& Sowa, D. (1986). Perceived organizational support. Journal of Applied Psychology, 71, 500-507.

Gemignani, J. (1997). When workers fight flab. Business and Health, 15, 39.

Goetzel, R., \& Ozminkowski, R. (2000). Health and productivity management: Emerging opportunities for health promotion professionals for the $21^{\text {st }}$ century. American Journal of Health Promotion, 14(4), 211-214.

Harris, J. S. (1994). The future of health promotion. In M. P. O'Donnell \& J. S. Harris (Eds.), Health promotion in the workplace, (pp. 525-543). New York: Delmar.

Highhouse, S., Levens, S., \& Sinar, E. F. (2003). Measuring attraction to organizations. Emotional and Psychological Measures, 63, 986-1001. 
Ji, S. H. (2003). Changes in the smoking rate and the social cost of smoking. Health and Welfare Policy Forum, 81, 16-24.

Kim, M. (October 14, 2008). SKT, 즐거운 사내펀드. 헤랄드 경제. [Online] Available: http://www.heraldbiz.com/SITE/data/html_dir/2008/10/14/200810140134.asp. (February 10, 2009)

Kim, Y.-M. (April 21, 2009). POSCO chief an agent of change at steelmaker. The Korea Herald. [Online] Available: http://www.koreaherald.co.kr/archives/result_contents.asp.

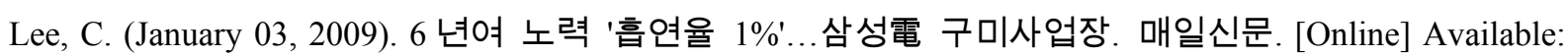
http://www.imaeil.com/sub_news/sub_news_view.php?news_id=399\&yy=2009. ( February 10, 2009)

Konovski, M. A., \& Cropanzano, R. (1991). Perceived fairness of employee drug testing as a predictor of employee attitudes and job performance. Journal of Applied Psychology, 76, 698-707.

Meritain Health. (2007). Healthy Merits. [Brochure]. [Online] Available: http://www.meritain.com/Home/BenefitSolutions/HealthyMerits. (January 10, 2008)

Osinubi, O. Y. O., Barbeau, E., Williams, J. M., \& Sorensen, G. (2005). Curbing the tobacco epidemic: The role of workplace tobacco control policies and programs. In J. H. Owing (Ed.), Focus on smoking and health research (pp. 1-75). New York: Nova.

Park, H. S. (2008). Centering in hierarchical linear modeling. Communication Methods and Measures, 2, 227-259.

Park, H. S., Dalsey, E., Kang, Y., Hong, S., \& Lee, S. A. (in press). Organizational attraction toward a company that adopts a smoke-free policy. Asia Pacific Journal of Management.

Pelletier, K. (1999). A review and analysis of the clinical and cost-effectiveness studies of comprehensive health promotion and disease management programs at the workplace: 1995 - 1998 update (IV). American Journal of Health Promotion, 13, 333-345.

PricewaterhouseCoopers. (2007). Behind the numbers: 2007 medical cost trends for employers. [Online] Available:

http://www.pwc.com/extweb/pwcpublications.nsf/docid/BBFCB2DC59B9C1CB85257261007A22C5.

Raudenbush, S. W., \& Bryk, A. S. (2002). Hierarchical linear models: Applications and data analysis methods (2nd ed.). Thousand Oaks, CA: Sage.

Rhoades, L., \& Eisenberger, R. (2002). Perceived organizational support: A review of the literature. Journal of Applied Psychology, 87, 698-714.

Schneider, B., Goldstein, H. W. \& Smith, D. B. (1995). The ASA framework: An update. Personnel Psychology, 48, 747-773.

Sofian, N. S., McAfee, T., Doctor, J., \& Carson, D. (1994). Tobacco control and cessation. In M. P. O'Donnell \& J. S. Harris (Eds.), Health promotion in the workplace (pp. 343-366). New York: Delmar.

Sugarman, S. D. (2003). Lifestyle" discrimination in employment. Berkeley Journal of Employment \& Labor Law, 24, 377-438.

Truxillo, D. M., Baier, T. N., \& Paronto, M. E. (2002). Reactions to organization alcohol testing and treatment programs. Journal of Business and Psychology, 17, 31-45.

Zoller, H. M. (2004). Manufacturing health: Employee perspectives o problematic outcomes in a workplace health promotion initiative. Western Journal of Communication, 68, 278-301. 
Table 1. Multilevel analyses results

\begin{tabular}{|c|c|c|c|c|c|}
\hline & Coefficient & $S E$ & $t$ & $d f$ & $p$-value \\
\hline \multicolumn{6}{|l|}{ For intercept $1, \beta_{0 j}$} \\
\hline Intercept $2, \gamma_{00}$ & 5.71 & 0.14 & 41.64 & 43 & $<.001$ \\
\hline Nation, $\gamma_{01}$ & -0.67 & 0.18 & -3.72 & 43 & .001 \\
\hline Perceived severity, $\gamma_{02}$ & 0.21 & 0.17 & 1.26 & 43 & .215 \\
\hline Perceived organizational support (POS), $\gamma_{03}$ & 0.31 & 0.11 & 2.67 & 43 & .011 \\
\hline \multicolumn{6}{|l|}{ For ex-smoker slope, $\beta_{1 j}$} \\
\hline Intercept $2, \gamma_{10}$ & -0.31 & 0.11 & -2.71 & 602 & .007 \\
\hline \multicolumn{6}{|l|}{ For non-smoker slope, $\beta_{2 i}$} \\
\hline Intercept $2, \gamma_{20}$ & -0.18 & 0.08 & -2.21 & 602 & .027 \\
\hline \multicolumn{6}{|l|}{ For perceived severity slope, $\beta_{3 j}$} \\
\hline Intercept $2, \gamma_{30}$ & -0.22 & 0.07 & -3.04 & 44 & .002 \\
\hline Nation, $\gamma_{31}$ & 0.15 & 0.08 & 1.98 & 44 & .053 \\
\hline Org. average POS, $\gamma_{32}$ & 0.1. & 0.05 & 2.61 & 44 & .013 \\
\hline \multicolumn{6}{|l|}{ For POS slope, $\beta_{4 j}$} \\
\hline Intercept $2, \gamma_{40}$ & 0.41 & 0.10 & 4.36 & 45 & $<.001$ \\
\hline Nation, $\gamma_{41}$ & -0.12 & 0.11 & -1.15 & 45 & .257 \\
\hline \multicolumn{6}{|l|}{ For ex-smoker $\times$ perceived severity slope, $\beta_{5 j}$} \\
\hline Intercept $2, \gamma_{50}$ & 0.21 & 0.08 & 2.43 & 602 & .016 \\
\hline \multicolumn{6}{|l|}{ For ex-smoker $\times$ POS slope, $\beta_{6 j}$} \\
\hline Intercept $2, \gamma_{60}$ & 0.14 & 0.09 & 1.58 & 602 & .115 \\
\hline \multicolumn{6}{|l|}{ For non-smoker $\times$ perceived severity slope, $\beta_{7 j}$} \\
\hline Intercept $2, \gamma_{70}$ & 0.13 & 0.06 & 2.12 & 602 & .034 \\
\hline \multicolumn{6}{|l|}{ For non-smoker $\times$ POS slope, $\beta_{8 j}$} \\
\hline Intercept $2, \gamma_{80}$ & 0.10 & 0.07 & 1.60 & 602 & .110 \\
\hline \multicolumn{6}{|l|}{ For perceived severity $\times$ POS slope, $\beta_{9 j}$} \\
\hline Intercept $2, \gamma_{90}$ & -0.02 & 0.03 & -0.61 & 602 & .545 \\
\hline
\end{tabular}

Note. Equations illustrating the model.

organizational attraction $_{i j}=\beta_{0 j}+\beta_{1 j}$ (ex-smoker) $+\beta_{2 j}$ (non-smoker $)+\beta_{3 j}$ (perceived severity) ${ }^{\#}+\beta_{4 j}$ (perceived organizational support $)^{\#}+\beta_{5 j}$ (ex-smoker $\times$ perceived severity) $+\beta_{6 j}$ (ex-smoker $\times$ perceived organizational support $)+\beta_{7 j}($ non-smoker $\times$ perceived severity $)+\beta_{8 j}($ non-smoker $\times$ perceived organizational support $)+r_{i j}$. $\beta_{0 j}=\gamma_{00}+\gamma_{01}\left(\right.$ nation $\left._{j}\right)+\gamma_{02}$ (org. average perceived severity $)^{*}+\gamma_{03}$ (org. average perceived organizational support $\left._{j}\right)^{*}+\mathrm{u}_{0 j}$

$\beta_{1 j}=\gamma_{10}$

$\beta_{2 j}=\gamma_{20}$,

$\beta_{3 j}=\gamma_{30}+\gamma_{31}$ (nation $\left._{j}\right)+\gamma_{32}$ (org. average perceived organizational support $)^{*}+\mathrm{u}_{3 j}$,

$\beta_{4 j}=\gamma_{40}+\gamma_{41}\left(\right.$ nation $\left._{j}\right)+u_{4 j}$,

$\beta_{5 j}=\gamma_{50}$,

$\beta_{6 j}=\gamma_{60}$,

$\beta_{7 j}=\gamma_{70}$,

$\beta_{8 j}=\gamma_{80}$

$\beta_{9 j}=\gamma_{90}$.

\# group-mean-centered

* grand-mean-centered

Ex-smoker: dummy-coded with smoker $=0$ and ex-smoker $=1$

Non-smoker: dummy-coded with smoker $=0$ and non-smoker $=1$

Nation: dummy-coded with USA $=0$ and Korea $=1$

Ex-smoker $\times$ perceived severity: an interaction term of dummy-coded ex-smoker variable by perceived severity Org. average perceived organizational support: organization average of perceived organizational support (i.e., individual employees' perceived organizational support scores were averaged to represent their corresponding organization's average) 